DR. DANIEL E FRANCÉS (Orcid ID : 0000-0003-4329-761X)

Article type : Liver Injury and Regeneration

\title{
Protective role of hepatocyte cyclooxygenase-2 expression against liver ischemia-reperfusion injury in mice
}

${ }^{\bullet}$ Omar Motiño, ${ }^{\bullet 2}$ Daniel E. Francés, ${ }^{1}$ Natalia Casanova, ${ }^{3}$ Marina Fuertes-Agudo, ${ }^{3}$ Carme Cucarella, ${ }^{4}$ Juana M. Flores, ${ }^{5}$ María Teresa Vallejo-Cremades, ${ }^{6}$ Luis Olmedilla, ${ }^{6}$ José Pérez Peña, ${ }^{6,7,9}$ Rafael Bañares, ${ }^{1,8,9}$ Lisardo Boscá, ${ }^{\bullet 3,8,9}$ Marta Casado and ${ }^{\bullet 1,8,9}$ Paloma Martín-Sanz.

\section{Affiliations}

${ }^{1}$ Instituto de Investigaciones Biomédicas (IIB) “Alberto Sols”, CSIC-UAM, Arturo Duperier 4, 28029 Madrid, Spain; ${ }^{2}$ Instituto de Fisiología Experimental (IFISE-CONICET), Suipacha 570, 2000 Rosario, Argentina; ${ }^{3}$ Instituto de Biomedicina de Valencia, IBV-CSIC, Jaume Roig 11, 46010 Valencia, Spain; ${ }^{4}$ Department of Animal Medicine and Surgery, Veterinary Faculty, Universidad Complutense de Madrid, 28040, Spain; ${ }^{5}$ Instituto de Investigación Hospital Universitario La Paz, IDIPAZ, Paseo de la Castellana 261, 28046 Madrid, Spain; ${ }^{6}$ Instituto de Investigación Sanitaria del Hospital Gregorio Marañón (IISGM), Dr. Esquerdo, 46, 28007 Madrid, Spain; ${ }^{7}$ Medicine Faculty, Universidad Complutense de Madrid, 28040 Madrid, Spain; ${ }^{8}$ Centro de Investigación Biomédica en Red de Enfermedades

Cardiovasculares (CIBERcv), Monforte de Lemos 3-5, 28029 Madrid, Spain; ${ }^{9}$ Centro de Investigación Biomédica en Red de Enfermedades Hepáticas y Digestivas (CIBERehd), Monforte de Lemos 3-5, 28029 Madrid, Spain.

This article has been accepted for publication and undergone full peer review but has not been through the copyediting, typesetting, pagination and proofreading process, which may lead to differences between this version and the Version of Record. Please cite this article as doi: 10.1002/hep.30241

This article is protected by copyright. All rights reserved. 


\section{- These authors contributed equally to this work}

\These two authors share senior authorship

Keywords: $\mathrm{COX}-2, \mathrm{PGE}_{2}$, liver, transplantation, hypoxia

- Corresponding authors: Dr. Paloma Martín-Sanz, Instituto de Investigaciones Biomédicas (IIB) “Alberto Sols”, CSIC-UAM. Madrid. Arturo Duperier, 428029 Madrid, Spain; Tel 34914972746, 34915854497 Fax: 34915854401; e-mail: pmartins@iib.uam.es or Dr. Marta Casado Pinna, Instituto de Biomedicina de Valencia (IBV-CSIC), Jaume Roig 11, 46010 Valencia; Tel +34963393778; Fax; 34963690800; e-mail: mcasado@ibv.csic.es.

List of Abbreviations: IRI, ischemia and reperfusion injury; ROS, reactive oxygen species; NF- $\kappa B$, nuclear factor- $\kappa B$; MPO, myeloperoxidase; NRF2, nuclear factor-erythroid 2-related factor 2; ERS, endoplasmic reticulum stress; LC3-I, microtubule associated protein 1 light chain 3; CHOP, C/EBP homologous protein; JNK, c-jun N-terminal kinase; IPC, ischemic preconditioning; COX-2, cyclooxygenase-2; $\mathrm{PGE}_{2}$, prostaglandin $\mathrm{E}_{2}$; hCOX-2-Tg, human COX-2 transgenic mice; Wt, wild type; DFU, (5,5-dimethyl-3(3-fluorophenyl)-4-(4methylsulfonyl)phenyl-2(5H)-furanone); ALT, alanine transaminase; AST, aspartate aminotransferase; LDH, lactate dehydrogenase; TNF- $\alpha$, tumor necrosis factor $\alpha$; IL-1 $\beta$, interleukin 1 $\beta$; IL-10, interleukin 10; IL-6, interleukin 6; Gpx, glutathione peroxidase; Sod1, cytosolic superoxide dismutase; Sod2, mitochondrial superoxide dismutase; DHE, dihydroethidium; ATG7, autophagy-related gene 7.

Financial Support: This work was supported by i-COOP2016-20213 (CSIC) to D.F., P.M.S; SAF2014-52492 (MINECO, Spain) to L.B.; CIBERcv and CIBERehd (ISCIII, Spain) to L.B., P.M.S and M.C.; SAF2016-75004-R (MINECO, Spain) to P.M.S. and M.C. M F-A. is 
recipient of FPI fellowship from MINECO (BES-2017-081928). We thank FEDER for financial support. Conflict of interest: The authors do not have any disclosures to report.

\section{ABSTRACT}

Liver ischemia and reperfusion injury (IRI) remains a serious clinical problem affecting liver transplantation outcomes. IRI causes up to $10 \%$ of early organ failure and predisposes to chronic rejection. Cyclooxygenase-2 (COX-2) is involved in different liver diseases but the significance of COX-2 in IRI is a matter of controversy. This study was designed to elucidate the role of COX-2 induction in hepatocytes against liver IRI. In the present work, hepatocytespecific COX-2 transgenic mice (hCOX-2-Tg) and their wild-type (Wt) littermates were subjected to IRI. hCOX-2-Tg mice exhibited lower grades of necrosis and inflammation than Wt mice, in part by reduced hepatic recruitment and infiltration of neutrophils, with a concomitant decrease in serum levels of pro-inflammatory cytokines. Moreover, hCOX-2-Tg mice showed a significant attenuation of the IRI-induced increase in oxidative stress and hepatic apoptosis, an increase in autophagic flux and a decrease in endoplasmic reticulum stress than in Wt mice. Interestingly, ischemic pre-conditioning of Wt mice resembles the beneficial effects observed in hCOX-2-Tg mice against IRI due to preconditioning-derived increase in endogenous COX-2, which is mainly localized in hepatocytes. Furthermore, measurement of $\mathrm{PGE}_{2}$ levels in plasma from patients who underwent liver transplantation revealed a significantly positive correlation of $\mathrm{PGE}_{2}$ levels and graft function, and an inverse correlation with the time of ischemia. Conclusion: These data support the view of a novel protective effect of hepatic COX-2 induction and the consequent rise of derived prostaglandins against IRI.

This article is protected by copyright. All rights reserved. 
Liver ischemia and reperfusion injury (IRI) remains a serious clinical problem affecting liver transplantation outcomes. IRI causes up to $10 \%$ of early organ failure and predisposes to chronic rejection (1). Moreover, the shortage of donor organs has led to an increased use of marginal livers, which are more susceptible to IRI (2). The mechanisms underlying hepatic ischemia and reperfusion injury are complex. However it is now becoming clear that reactive oxygen species (ROS) activate redox-regulated transcription factors such as nuclear factor- $\kappa \mathrm{B}$ $(\mathrm{NF}-\kappa \mathrm{B})$, which trigger the secretion of pro-inflammatory cytokines, lead to tissue injury $(3,4)$. In response to these cytokines, neutrophils are recruited into the injured tissue and they further release ROS, cytokines, myeloperoxidase (MPO) and other mediators which aggravate tissue damage $(5,6)$. Moreover, oxidative stress induces the translocation of the nuclear factor-erythroid 2-related factor (NRF2), and the expression of several antioxidant and detoxification enzymes, such as glutathione-S-transferases, quinine oxidoreductase 1, glutamate cysteine ligase and heme oxygenase 1 , which in turn decrease ROS and reduce oxidative stress (7).

Additionally, IRI impairs autophagy (8) and activates endoplasmic reticulum stress (ERS) $(6,9)$. Autophagy is a catabolic process mainly regulated by the conjugation of microtubule associated protein 1 light chain 3 (LC3-I) to the membrane lipid phosphatidylethanolamine to form LC3-II. LC3-II serves as a recognition site for LC3-binding chaperones such as p62 that deliver their cargo to autophagosomes (10). Then, autophagosomes reach lysosomes and fuse to form autophagolysosomes, allowing the degradation by lysosomal acid hydrolases of their contents (11). ERS, initiated by a defensive process known as the unfolded protein response (UPR), comprises cellular mechanisms for adaptation of cell survival characterized by translational arrest of protein synthesis. UPR is mediated by the release of intraluminal chaperone glucose-regulated protein 78 (GRP78) from the ER sensors (PKR-like eukaryotic 
initiation factor 2 kinase (PERK), activating transcription factor 6 (ATF-6), and the inositol requiring $1 \alpha(\operatorname{IRE} 1 \alpha)$. If the cell fails to adapt, oxidative and inflammatory signaling pathways are activated leading to apoptosis through PERK-mediated C/EBP homologous protein $(\mathrm{CHOP})$ expression and signal-regulated kinase 1/C-jun N-terminal kinase (JNK), among others (12).

In the liver, and in various tissues, short periods of ischemia protect efficiently against subsequent IRI. This phenomenon, known as ischemic preconditioning (IPC), indicates that a brief ischemic insult triggers a protective biological reaction in the liver which is associated with inhibition of pro-apoptotic pathways (13).

Cyclooxygenase (COX)-1 and -2 catalyze the first step in prostanoid biosynthesis. COX-1 is constitutively expressed in many tissues, whereas COX-2 is induced by a variety of stimuli (14) and its expression has been detected in several liver pathologies (15). In the other hand, hepatocyte-specific constitutive expression of COX-2 exerts an efficient protection against acute liver injury by an antiapoptotic/antinecrotic effect (16). Controversial results have been published related to COX-2 and IRI. It was reported that several COX-2 inhibitors protect mice and rats from hepatic IRI (17-19). However, Kuzumoto et al. (20) reported that the prostaglandin $\mathrm{E}_{2}\left(\mathrm{PGE}_{2}\right)-\mathrm{EP}_{4}$ signaling pathway has an inhibitory role in hepatic IRI, showing that $\mathrm{EP}_{4}$ agonists effectively protect against IRI. Since there is a lack of studies on the effects of hepatic COX-2 induction and prostaglandins during liver IRI, in the present study we have demonstrated that constitutive hepatocyte expression of COX-2 significantly protects against IRI by decreasing inflammation, oxidative stress, apoptosis, and ERS. Moreover, the constitutive hepatocyte expression of COX-2 increase survival and autophagic signaling pathways, suggesting a novel cytoprotective role for COX-2 in hepatic IRI.

This article is protected by copyright. All rights reserved. 


\section{EXPERIMENTAL PROCEDURES}

\section{Patients}

Arterial $\mathrm{PGE}_{2}$ plasma concentration in a cohort of 63 human liver transplants from cadaveric donors, 60 min after reperfusion, was analyzed. This analysis was approved by the Ethics Committee on Clinical Research of the University General Hospital Gregorio Marañón (Madrid, Spain, June 2016). All the patients had already signed a previous informed consent allowing the plasma analysis. Early graft function was assessed by the Toronto classification that accounts for elevation of ALT, coagulopathy and bile flow (21). Patients were classified as good early graft function (grades I and II) and bad early graft dysfunction (grades III and IV). Graft ischemia time was recorded in all cases.

\section{Animal experimentation}

Male twelve-weeks-old COX-2 transgenic mice (hCOX-2-Tg) and their wild-type (Wt) siblings were used. The mice were divided randomly into four groups: 1) Sham operation (Sh) group; 2) hepatic ischemia-reperfusion (I/R) group; 3) ischemic preconditioning (Wt PC) group; and 4) 5,5-dimethyl-3(3-fluorophenyl)-4-(4-methylsulfonyl)phenyl-2(5H)furanone (DFU) treated group (Wt PC DFU and hCOX-2-Tg DFU). Animals were anesthetized with isoflurane $1.2-2 \%$ and a model of segmental (70\%) warm hepatic ischemiareperfusion protocol was assessed (22). Reperfusion was initiated by removal of the clamp for $4 \mathrm{~h}$ and $24 \mathrm{~h}$. For IPC experiments, Wt mice were subjected to $20 \mathrm{~min}$ of ischemia followed by $30 \mathrm{~min}$ of reperfusion prior to $90 \mathrm{~min}$ of ischemia and $4 \mathrm{~h}$ of reperfusion. For DFU treatment, Wt PC and hCOX-2-Tg mice were injected intraperitoneally with $5 \mathrm{mg} / \mathrm{kg}$ DFU in DMSO three days and $45 \mathrm{~min}$ before the PC or hepatic ischemia, respectively. Control mice were treated with DMSO. The animals were sacrificed and livers were removed and rapidly snap-frozen in liquid nitrogen and stored at $-80^{\circ} \mathrm{C}$, collected in a solution

This article is protected by copyright. All rights reserved. 
containing $30 \%$ sucrose in PBS or fixed in $10 \%$ buffered formalin. Plasma was obtained from the inferior cava vein. All animal experimentation was controlled following the recommendations of the Federation of European Laboratory Animal Science Associations (FELASA) on health monitoring, European Community Law (2010/63/UE) and the Spanish law (R.D. 53/2013) with approval of the Ethics Committee of the Spanish National Research Council, Spain.

\section{Whole transcriptome analysis}

Total RNA was obtained using the mirVana ${ }^{\mathrm{TM}}$ miRNA Isolation Kit (Thermo Fisher Scientific, Waltham, MA USA), prepared for sequencing using the Illumina TruSeq RNA Sample Prep Kit v2 according to the manufacturer's instructions, and sequenced on a Hiseq 4000 platform (Macrogen, Geumchcon-gu, Seoul, Korea). The Illumina Hiseq 4000 generated raw images utilizing HCS (HiSeq Control Software v3.3) for system control and base calling through an integrated primary analysis software called RTA (Real Time Analysis. v2.7.3). The BCL (base calls) binary was converted into FASTQ utilizing illumina package bcl2fastq (v2.17.1.14). Trimmed 100-bp sequenced paired-end reads were mapped to reference genome with HISAT2. After the read mapping, Stringtie was used for transcript assembly. Expression profile was calculated for each sample and transcript/gene as FPKM (Fragment per Kilobase of transcript per Million mapped reads).

\section{Data analysis.}

Data are expressed as means \pm S.E. Statistical significance was tested by one-way ANOVA, followed by Bonferroni's test. Analysis was performed by using the statistical software GraphPad Prism 5. For patients, the Kolmogorov-Smirnov test was used to assess the normality of the distribution of numeric variables. To compare means between independent 
samples, Mann-Whitney rank-sum test were used. To assess a significant relationship between two variables, Spearman's correlation was calculated. Data were analyzed using SPSS IBM for Windows statistical package, version 24 (SPSS Inc, Chicago, IL, USA). For all statistical tests, a $P$ value $<0.05$ was considered significant.

\section{RESULTS}

\section{Hepatic COX-2 expression protects transgenic mice from IRI-induced liver damage.}

We used our previously described transgenic mice model, hCOX-2-Tg, that constitutively expresses human COX-2 in hepatocytes under the control of the human APOE promoter containing the hepatic control region (HCR) (23). The expression of hCOX-2 in these livers is comparable to the endogenous levels reached in regenerating liver after partial hepatectomy (24). No changes in COX-2 mRNA or protein levels were found in hCOX-2-Tg mice after 90 min of ischemia and $4 \mathrm{~h}$ or $24 \mathrm{~h}$ of reperfusion (Supplementary Fig. 1B and Fig. 1A). Furthermore, Cox-1 mRNA and COX-1 protein levels were maintained without changes in all conditions (Supplementary Fig. 1A and Fig. 1A).

$\mathrm{PGE}_{2}$ levels were, as expected, significantly higher in hCOX-2-Tg when compared to $\mathrm{Wt}$ mice, even when there is a clear tendency to increase $\mathrm{PGE}_{2}$ hepatic levels in Wt mice $24 \mathrm{~h}$ post IRI (Fig. 1B). This increase is associated to an induction of endogenous COX-2 expression (Fig. 6E). Liver sections were stained with hematoxylin/eosin and then evaluated by an experienced liver pathologist. Extensive parenchymal areas of necrosis and vascular congestion were observed in Wt mice at $4 \mathrm{~h}$ and $24 \mathrm{~h}$ after IRI. Necrotic hepatocytes were characterized by condensation of chromatin, swelling of organelles, karyolysis and cell rupture. These pathological features were significantly attenuated in hCOX-2-Tg hepatocytes (Fig. 1C).

This article is protected by copyright. All rights reserved. 
Elevated serum levels of aminotransferases such as alanine transaminase (ALT) and aspartate aminotransferase (AST) reflect the degree of hepatic cell damage. As shown in Fig. 1D, IRI resulted in a marked elevation of serum ALT and AST levels, but they were significantly lower in hCOX-2-Tg mice after $4 \mathrm{~h}$ of reperfusion. Lactate dehydrogenase (LDH) activity was measured as necrotic marker (25). The increase in LDH activity after IRI was much lower in hCOX-2-Tg mice at $4 \mathrm{~h}$ and $24 \mathrm{~h}$ (Fig. 1D), confirmed by the quantification of the necrosis grade (Fig. 1E).

\section{Alteration of gene expression profile after ischemia/reperfusion injury.}

Murine whole transcriptome sequencing was performed in order to examine the different gene expression profiles in the hCOX-2 Tg mice after IRI (90 min ischemia, $4 \mathrm{~h}$ reperfusion). A total of 70 genes showed significant differential expression (false discovery rate $\leq 0.05$, $\log _{2} \geq 2$; Fig. 2A). Among these genes, 18 genes were up-regulated and 52 genes were downregulated (Fig. 2B). To elucidate the roles of differentially expressed genes, GO (gene ontology) analysis was used to analyze the biological functions of those genes (Fig. 2C and Supplementary Table 4). The results indicated that several pathways were consistently modified after IRI between mice models, such as biological processes related with the response to stress and inflammation. Taking into account that inflammation and oxidant stress are the disease mechanisms most commonly implicated in hepatic IRI $(5,6)$, we decided to validate and to study in depth these two pathways.

\section{COX-2 expression reduced hepatic inflammation in the I/R injury model.}

Pro-inflammatory cytokines such as tumor necrosis factor $\alpha$ (TNF- $\alpha$ ) and interleukin $1 \beta$ (IL$1 \beta)$ are released by activated Kupffer cells, and induce hepatocellular and endothelial injuries. Furthermore, the NLRP3 inflammasome controls caspase-1 activity, IL-1 $\beta$ release and is 
activated in IRI, by ROS production (26). Constitutive COX-2 expression reduced the hepatic mRNAs levels of these pro-inflammatory cytokines including Nlrp3 inflammasome. However, interleukin 10 (Il-10) expression, an anti-inflammatory interleukin, was increased in hCOX-2-Tg mice. (Fig. 3A). Consistently, plasmatic levels of IL-1 $\beta$, interleukin 6 (IL-6) and TNF- $\alpha$ reached up the higher levels at $24 \mathrm{~h}$ of reperfusion, while they were significantly lower in hCOX-2-Tg vs. Wt (Fig. 3B). Our previous data showed lower levels of inflammatory markers and reduced hepatic leukocyte recruitment and infiltration in hCOX-2$\mathrm{Tg}$ mice upon different liver injuries $(16,27)$. There was no evidence of neutrophil accumulation after $4 \mathrm{~h}$ of reperfusion. Tissue MPO activity, an index of neutrophil infiltration, was found increased at $24 \mathrm{~h}$ of reperfusion in Wt mice (Fig. 3C). Additionally, at this time point, immunohistochemistry analysis showed lesser levels of infiltrating $\mathrm{Ly}_{6 \mathrm{G}}{ }^{+}$ cells (a neutrophil plasma membrane biomarker) when comparing hCOX-2-Tg vs. Wt IR groups (Fig. 3D).

$\mathrm{NF}-\kappa \mathrm{B}$ is not only an important mediator of the inflammatory response but its signaling pathway is a key component in the progression of IR derived injury (28). To know whether $\mathrm{NF}-\kappa \mathrm{B}$ is involved in the regulation of inflammation in our model, nuclear p65 and phosphorylated cytosolic I $\mathrm{I} \mathrm{B} \alpha$ protein levels were analyzed by Western blot in $\mathrm{Wt}$ and hCOX-2-Tg liver subjected to IRI. We found in Wt IRI mice an important increase in nuclear p65 localization and in $\mathrm{pI \kappa} B \alpha$ protein levels in hepatocytes, suggesting a strong $\mathrm{NF}-\kappa \mathrm{B}$ pathway activation, whereas this effect resulted attenuated in hCOX-2-Tg mice (Fig. 3E-F).

\section{Constitutive hepatocyte COX-2 expression leads to decreased apoptosis and to an increase}

\section{in antioxidant response.}

The apoptotic process is a consequence of reperfusion, which marks the onset and progression of hepatocellular damage (29). We described previously an anti-apoptotic role for 
hepatic COX-2 expression $(23,30)$; to further explore the protective effect of $\mathrm{COX}-2$, caspase-3 activity and some key apoptotic/anti-apoptotic protein levels were measured. As shown in supplementary Fig. 2A, caspase-3 activity increased only in Wt mice after IRI. Moreover, an important increase in the BCL-2-associated X protein/ B-cell lymphoma 2 (BAX/BCL-2) ratio was found in Wt IRI mice at $24 \mathrm{~h}$ of reperfusion. This effect was attenuated in hCOX-2-Tg IRI mice (Supplementary Fig. 2B). Additionally, after IRI an increase in $\mathrm{p}-\mathrm{AKT} / \mathrm{AKT}$ and in $\mathrm{p}-\mathrm{AMPK} \alpha / \mathrm{AMPK} \alpha$ ratios were observed in hCOX-2-Tg when compared to Wt mice (Supplementary Fig. 2C). DFU treatment reversed the increase in p-AKT/AKT and in p-AMPKa/AMPK $\alpha$ ratios (data not shown).

IRI is associated with the generation of ROS and other oxidative stress-related molecules. In this regard, some COX-2-derived prostanoid actions were associated to an enhanced antioxidant response (31). The expression of several antioxidant genes is shown in Fig. 4A. The mRNA levels of $N f r 2$, heme oxygenase 1 (Hmoxl), glutathione peroxidase (Gpx), glutathione reductase $(G s r)$, cytosolic superoxide dismutase Sod1 and mitochondrial Sod2, were increased in hCOX-2-Tg mice after $4 \mathrm{~h}$ of IRI vs. Wt IRI. To gain insight into the mechanisms involved, nuclear extracts were prepared to quantify the protein levels of NRF2. Nuclear localization of NRF2 increased in hCOX-2-Tg after IRI (Fig. 4B), and mainly hepatocyte location was corroborated by immunofluorescence staining (Fig. 4C). In this sense, we use albumin as a specific hepatocyte marker, and CLEC4F as Kupffer cell marker (32). Moreover, the activities of antioxidants enzymes were measured and there was an increase in MnSOD, GSR and GPX activities at $4 \mathrm{~h}$ in hCOX-2-Tg mice after IRI (Fig. 4D and Supplementary Table 1). Indeed, MnSOD protein levels were already increased in hepatocytes from hCOX-2-Tg in sham conditions. This difference was maintained at $4 \mathrm{~h}$ or 24 h of IRI (Supplementary Fig. 3A-B).

This article is protected by copyright. All rights reserved. 
Dihydroethidium (DHE) staining was performed as an indirect measurement of ROS production. ROS levels were significantly increased in Wt IRI condition at $4 \mathrm{~h}$ (Fig. 4E and Supplementary Fig. 3C). These results were confirmed by the measurement of lipid peroxidation (LPO) levels. LPO increased by IRI only in livers of Wt mice (Supplementary Fig. 3D) suggesting a diminished ROS production in hCOX-2-Tg mice. In line with this, total glutathione content was decreased after IRI, but to a lesser extent in hCOX-2-Tg. Also, the ratio of oxidized/total glutathione (GSSG/GSHt), evaluated as an intracellular redox status marker, was significantly higher in Wt IRI mice after $4 \mathrm{~h}$ (Fig. 4F). Overall, these data are compatible with an attenuated oxidative stress in hCOX-2-Tg IRI mice when compared to $\mathrm{Wt}$ IRI.

\section{Hepatocyte COX-2 expression attenuates endoplasmic reticulum stress and activates} autophagy after IRI.

It has been suggested that impaired autophagy increased hepatocellular damage during the reperfusion period. Therefore, we analyzed p62 and LC3-II protein levels. As shown in Fig. 5A-C, p62 protein levels were decreased in hCOX-2-Tg after IRI whereas LC3-II and autophagy-related gene 7 (ATG7) levels increased. Overall, these data suggest an improvement of the autophagic process in hCOX-2-Tg liver after IRI. This effect was also observed using electron microscopy since we detected more double membrane structures (autophagosomes) in hCOX-2-Tg than in Wt after IRI (Fig. 5D).

Next, we examined if the changes in the autophagic process found during IRI were accompanied by activation of the ERS signaling pathway. Significant increases of GRP78 (at $24 \mathrm{~h}$ ), CHOP (at $4 \mathrm{~h}$ and $24 \mathrm{~h}$ ) and p-JNK (at $4 \mathrm{~h}$ ) were found in Wt mice after IRI vs. the corresponding hCOX-2-Tg mice (Fig. 5E-F and Supplementary Fig. 2C) suggesting an 
attenuated activation of the ERS signaling in these mice livers. Moreover, DFU treatment, increased JNK phosphorylation in hCOX-2-Tg mice (data not shown).

\section{Ischemic preconditioning attenuated I/R damage in Wt mice and was associated to}

\section{endogenous COX-2 induction.}

To check the possible role of endogenous $\mathrm{COX}-2$ induction in the protective effect observed after IRI, we performed IPC in the Wt mice and inhibition of COX-2 activity in Wt IPC and hCOX-2-Tg mice with the COX-2 specific inhibitor DFU. When compared to Wt IRI, Wt IPC exhibited a decrease in the necrosis grade and vascular congestion, whereas DFU treatment led to a significantly higher necrosis grade in Wt IPC and hCOX-2-Tg IRI mice (Fig. 6A-B). These effects were reflected in the plasma levels of ALT and AST and LDH in accordance with the necrosis grade (Fig. 6C). Consistently, increased levels of $\mathrm{PGE}_{2}$ were observed in the Wt IPC group and in hCOX-2-Tg mice when compared to Wt IRI animals, while DFU decreased these levels in both conditions (Fig. 6D). Higher $\mathrm{PGE}_{2}$ levels were associated with more pronounced changes in mRNA and protein levels of endogenous COX2 in Wt IPC $v s$ Wt mice after IRI (Fig. 6E). Moreover, we confirmed that endogenous COX-2 induced-expression is mainly in the hepatocytes (Fig. 6F). These data confirm the specificity of COX-2-dependent $\mathrm{PGE}_{2}$ involvement in the protective effect.

To assess the contribution of endogenous COX-2 to the protection against IRI, the expression profile of inflammatory and antioxidants genes was analyzed and, as shown in Fig. 7A, IPC decreased Il-1b, Il-6, Tnfa and Nlrp3 expression and increased Il-10 in Wt IPC when compared to Wt IRI, resembling the constitutive COX-2 expression phenotype. The expression of antioxidants genes was increased in Wt mice subjected to IPC surgery; however, DFU treatment had no effect over these genes probably because the decrease in $\mathrm{PGE}_{2}$ was not sufficient to suppress the modulation of these antioxidant genes (Fig. 7B).

This article is protected by copyright. All rights reserved. 


\section{Elevated plasmatic PGE $E_{2}$ levels correlates with an improvement of liver function after LT.}

To evaluate the contribution of $\mathrm{PGE}_{2}$ dynamics in a clinical setting, we analyzed arterial plasma $\mathrm{PGE}_{2}$ concentration in a cohort of 63 patients after receiving a liver transplant (LT). Arterial $\mathrm{PGE}_{2}$ levels 60 min after reperfusion were significantly higher in patients with good graft function as compared in patients with poor graft function (479 (335-748), vs. 276 (148579) $\mathrm{pg} / \mathrm{ml}$ ) (Fig. 8A). Additionally, there was a significant inverse correlation between $\mathrm{PGE}_{2}$ levels and graft ischemia time (Fig. 8B) as well as with the time of hospital stay (Fig. 8C).

\section{DISCUSSION}

We used hCOX-2-Tg mice to investigate the role of COX-2 in the outcome of liver after IRI. Our data demonstrate that hCOX-2-Tg mice were significantly less susceptible to liver IRI. hCOX-2-Tg mice showed reduced ALT, AST and LDH levels and necrosis grade after IRI, which indicates a lesser liver damage when compared to Wt animals. Moreover, IPC attenuated IRI damage in Wt mice through the induction of endogenous COX-2 expression, which is mainly localized in hepatocytes. Our observation that expression of COX-2 confers a protective role in liver suffering IRI is supported by the fact that DFU treatment restores most of the IRI parameters both in Wt IPC and hCOX-2 Tg mice. These results are also supported by other publications, in which COX-2 expression was beneficial in rat liver IRI $(20,33)$, and in the immediate human postsurgical liver transplantation period (34).

Consistent with previous reports $(16,27)$ constitutive hepatic COX-2 expression leads to lower plasmatic levels of pro-inflammatory cytokines (i.e. IL-1 $\beta$, IL-6 and TNF- $\alpha$ ). hCOX-2$\mathrm{Tg}$ mice presented an attenuated inflammatory response with lower levels of proinflammatory markers, and higher levels of IL-10, leading to a diminished hepatic IRI. Our in vivo data support: a) an attenuated inflammatory response, at least in part, by decreasing IRI- 


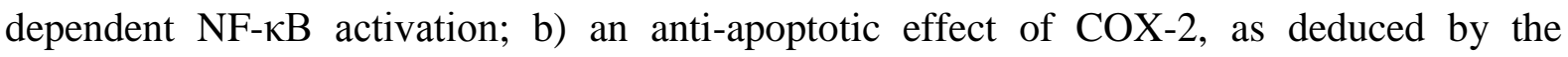
decrease of caspase-3 activation after IRI; and c) an improved antioxidant response to oxidative stress and decreased ROS production induced by COX-2 after IRI.

Interestingly, Hamada et al. (35) reported that global COX-2 deficiency enhances Th2 immune function and impairs neutrophil recruitment in liver IRI. Nevertheless, the same authors reported that selective deletion of myeloid cell derived COX-2 failed to ameliorate liver IRI and developed hepatic extensive necrosis and leukocyte infiltration (36). These authors concluded that selective myeloid COX-2 gene inactivation, which is the major source of COX-2 activity in non-parenchymal hepatic cells, has no effect in the progression of liver damage in response to IRI. However, inhibition of inducible $\mathrm{PGE}_{2}$ synthase attenuates reperfusion injury through inactivation of Kupffer cells and neutrophils, indicating a crucial role for PGs in hepatic IRI (37). In our experimental model, the results clearly support that constitutive expression of COX-2 in hepatic parenchymal cells lead to a diminished neutrophil infiltration in liver after IRI as indicated by $\mathrm{Ly} 6 \mathrm{G}^{+}$immunostaining and MPO activity.

In hepatic IRI, a dead liver cell is usually present in a necrotic form but malfunctioning programmed cell death is also observed in the hepatic IRI process (38). We found an attenuated response in BAX/BCL-2 ratio and caspase 3 activity in hCOX-2-Tg mice after IRI in agreement with the reported anti-apoptotic effect of COX-2 activity $(16,27)$.

The phosphatidylinositide 3-kinase/AKT and AMPK pathways play a central role in integrating diverse survival signals. It is known that the AKT pathway is target of prostaglandins and that AKT phosphorylation is enhanced in the liver of hCOX-2-Tg reinforcing the survival pathways $(30,39)$. The increase in the p-AKT/AKT ratio in hCOX-2- 
Tg mice after IRI may be due to a direct AKT activation through COX-2-dependent $\mathrm{PGE}_{2}$ acting via EP2/EP4 G $\beta \gamma$ dimers, as reported by Rizzo et al.(40). It has been described that the activation of both AKT and AMPK is part of a protective mechanism to prevent IRI (41). Our results agree with this notion, showing not only an increase in p-AKT/AKT ratio, but also in the p-AMPK to AMPK ratio in hCOX-2-Tg mice after IRI.

Ke et al. have reported that NRF2 has a protective role against hepatocellular damage induced by ischemia (42). In fact, pre-treatment with a NRF2 inducer resulted in partial attenuation of hepatic IRI in rats (7). It has been described that some COX-2 metabolites can induce a nuclear translocation of NRF2 (43) and even reduce ROS levels in a model of IRI (31). Our results show a clear induction of Nrf2, Hmoxl, Sodl and Sod2 expression in hCOX2-Tg mice after IRI when compared to Wt with a concomitant increase in the nuclear protein levels of NRF2 and in the activities of antioxidant enzymes, hence, leading to an induction of antioxidant response. This pattern was mimicked by IPC in Wt mice.

Several reports have suggested that IRI is characterized by defective liver autophagy (8). In this work we show an impaired autophagic flux after IRI in Wt mice. The-decreased autophagy was attenuated in hCOX-2-Tg IRI in agreement with previous works that reported in other cells an autophagy induction via up-regulation of COX-2 and $\mathrm{PGE}_{2}$ production (44), or that mPGES-2 down regulation is associated with autophagy inhibition and enhanced apoptosis (45).

Recent experimental data suggest the involvement of endoplasmic reticulum stress (ERS) in several liver diseases, including IRI (46). As a proof of concept, chemical chaperones such as 4-phenylbutyrate are able to protect against IRI by inhibition of ERS (47). Our data show that 
after IRI, in Wt mice, an impaired autophagy associated with increased ERS after IRI occurs, involving an increase in ERS markers, such as GRP78, CHOP and phosphorylated JNK. Again, constitutive hepatocyte COX-2 expression attenuates ERS and activates autophagy after IRI.

From a translational point of view, we evaluated the relationship between $\mathrm{PGE}_{2}$ levels in plasma and the clinical data of patients undergoing liver transplantation (LT) (48). Interestingly, clinical data obtained in the context of LT showed a significant association between arterial $\mathrm{PGE}_{2}$ levels and early graft function, evaluated by a well-known scale that has been employed in previous publications (49). Additionally, ischemia time was also inversely associated to $\mathrm{PGE}_{2}$. These results are clearly in consonance with the experimental results obtained in transgenic animals in which overexpression of COX-2 has protective effect in several experimental settings. Our results suggest that, even in the physiological range levels, prostaglandins may have a protective role in patients receiving liver transplantation.

Overall, the data support beneficial and protective effects of hepatocyte COX-2 induction and its dependent prostaglandins during IPC as a key hepatic protective mechanism triggered against I/R-derived damage and might provide evidence-based support for further therapeutic interventions after liver injury.

This article is protected by copyright. All rights reserved. 


\section{REFERENCES}

1. Casillas-Ramírez A, Mosbah IB, Ramalho F, Roselló-Catafau J, Peralta C. Past and future approaches to ischemia-reperfusion lesion associated with liver transplantation. Life Sci. 2006;79:1881-1894.

2. Busuttil RW, Tanaka K. The utility of marginal donors in liver transplantation. Liver Transpl. 2003;9:651-663.

3. Jaeschke H. Reactive oxygen and mechanisms of inflammatory liver injury: Present concepts. J Gastroenterol Hepatol. 2011;26 Suppl 1:173-179.

4. Yu J, Lee H-S, Lee S-M, Yu H-C, Moon WS, Chung MJ, et al. Aggravation of postischemic liver injury by overexpression of A20, an NF-kB suppressor. J Hepatol. 2011;55:328-336.

5. Jaeschke H. Mechanisms of Liver Injury. II. Mechanisms of neutrophil-induced liver cell injury during hepatic ischemia-reperfusion and other acute inflammatory conditions. Am J Physiol Gastrointest Liver Physiol. 2006;290:G1083-8.

6. Elias-Miró M, Jiménez-Castro MB, Rodés J, Peralta C. Current knowledge on oxidative stress in hepatic ischemia/reperfusion. Free Radic Res. 2013;47:555-568.

7. Masuda Y, Vaziri ND, Takasu C, Li S, Robles L, Pham C, et al. Salutary effect of pretreatment with an Nrf2 inducer on ischemia reperfusion injury in the rat liver. Gastroenterol Hepatol (Que). 2014;1:1-7.

8. Liu A, Huang L, Guo E, Li R, Yang J, Li A, et al. Baicalein pretreatment reduces liver ischemia/reperfusion injury via induction of autophagy in rats. Sci Rep. 2016;6:25042.

9. González-Rodríguez A, Mayoral R, Agra N, Valdecantos MP, Pardo V, MiquilenaColina ME, et al. Impaired autophagic flux is associated with increased endoplasmic reticulum stress during the development of NAFLD. Cell Death Dis. 2014;5:e1179.

10. Komatsu M, Ichimura Y. Physiological significance of selective degradation of p62 by 
autophagy. FEBS Lett. 2010;584:1374-1378.

11. Murrow L, Debnath J. Autophagy as a stress-response and quality-control mechanism: implications for cell injury and human disease. Annu Rev Pathol. 2013;8:105-137.

12. Hotamisligil GS. Endoplasmic reticulum stress and the inflammatory basis of metabolic disease. Cell. 2010;140:900-917.

13. Selzner N, Rudiger H, Graf R, Clavien P-A. Protective strategies against ischemic injury of the liver. Gastroenterology. 2003;125:917-936.

14. Ricciotti E, FitzGerald GA. Prostaglandins and inflammation. Arterioscler Thromb Vasc Biol. 2011;31:986-1000.

15. Fernández-Alvarez A, Llorente-Izquierdo C, Mayoral R, Agra N, Boscá L, Casado M, et al. Evaluation of epigenetic modulation of cyclooxygenase-2 as a prognostic marker for hepatocellular carcinoma. Oncogenesis. 2012;1:e23.

16. Mayoral R, Mollá B, Flores JM, Boscá L, Casado M, Martín-Sanz P. Constitutive expression of cyclo-oxygenase 2 transgene in hepatocytes protects against liver injury. Biochem J. 2008;416:337-346.

17. Fu H, Chen H, Wang C, Xu H, Liu F, Guo M, et al. Flurbiprofen, a cyclooxygenase inhibitor, protects mice from hepatic ischemia/reperfusion injury by inhibiting GSK-3 $\beta$ signaling and mitochondrial permeability transition. Mol Med. 2012;18:1128-1135.

18. Tolba RH, Fet N, Yonezawa K, Taura K, Nakajima A, Hata K, et al. Role of preferential cyclooxygenase-2 inhibition by meloxicam in ischemia/reperfusion injury of the rat liver. Eur Surg Res. 2014;53:11-24.

19. Zhang T, Ma Y, Xu K-Q, Huang W-Q. Pretreatment of parecoxib attenuates hepatic ischemia/reperfusion injury in rats. BMC Anesthesiol. 2015;15:165.

20. Kuzumoto Y, Sho M, Ikeda N, Hamada K, Mizuno T, Akashi S, et al. Significance and therapeutic potential of prostaglandin E2 receptor in hepatic ischemia/reperfusion

This article is protected by copyright. All rights reserved. 
injury in mice. Hepatology. 2005;42:608-617.

21. Greig PD, Woolf GM, Sinclair SB, Abecassis M, Strasberg SM, Taylor BR, et al. Treatment of primary liver graft nonfunction with prostaglandin E1. Transplantation. $1989 ; 48: 447-453$.

22. Selzner N, Selzner M, Jochum W, Clavien P-A. Ischemic preconditioning protects the steatotic mouse liver against reperfusion injury: an ATP dependent mechanism. J Hepatol. 2003;39:55-61.

23. Casado M, Mollá B, Roy R, Fernández-Martínez A, Cucarella C, Mayoral R, et al. Protection against Fas-induced liver apoptosis in transgenic mice expressing cyclooxygenase 2 in hepatocytes. Hepatology. 2007;45:631-638.

24. Casado M, Callejas NA, Rodrigo J, Zhao X, Dey SK, Boscá L, et al. Contribution of cyclooxygenase 2 to liver regeneration after partial hepatectomy. FASEB J. 2001;15:2016-2018.

25. Chan FK-M, Moriwaki K, De Rosa MJ. Detection of necrosis by release of lactate dehydrogenase activity. Methods Mol Biol. 2013;979:65-70.

26. Kim H-Y, Kim S-J, Lee S-M. Activation of NLRP3 and AIM2 inflammasomes in Kupffer cells in hepatic ischemia/reperfusion. FEBS J. 2015;282:259-270.

27. Motiño O, Agra N, Brea Contreras R, Domínguez-Moreno M, García-Monzón C, Vargas-Castrillón J, et al. Cyclooxygenase-2 expression in hepatocytes attenuates nonalcoholic steatohepatitis and liver fibrosis in mice. Biochim Biophys Acta. 2016;1862:1710-1723.

28. He D, Guo Z, Pu J-L, Zheng D-F, Wei X-F, Liu R, et al. Resveratrol preconditioning protects hepatocytes against hepatic ischemia reperfusion injury via Toll-like receptor 4/nuclear factor- $\kappa \mathrm{B}$ signaling pathway in vitro and in vivo. Int Immunopharmacol. 2016;35:201-209.

This article is protected by copyright. All rights reserved. 
29. Chang WJ, Chehab M, Kink S, Toledo-Pereyra LH. Intracellular calcium signaling pathways during liver ischemia and reperfusion. J Invest Surg. 2010;23:228-238.

30. Francés DEA, Ingaramo PI, Mayoral R, Través P, Casado M, Valverde ÁM, et al. Cyclooxygenase-2 over-expression inhibits liver apoptosis induced by hyperglycemia. $\mathrm{J}$ Cell Biochem. 2013;114:669-680.

31. Chen K, Li J-J, Li S-N, Feng J, Liu T, Wang F, et al. 15-Deoxy- $\Delta 12,14$-prostaglandin J2 alleviates hepatic ischemia-reperfusion injury in mice via inducing antioxidant response and inhibiting apoptosis and autophagy. Acta Pharmacol Sin. 2017;38:672-687.

32. Scott CL, Zheng F, De Baetselier P, Martens L, Saeys Y, De Prijck S, et al. Bone marrow-derived monocytes give rise to self-renewing and fully differentiated Kupffer cells. Nat Commun. 2016;7:10321.

33. Hossain MA, Wakabayashi H, Izuishi K, Okano K, Yachida S, Maeta H. The role of prostaglandins in liver ischemia-reperfusion injury. Curr Pharm Des. 2006;12:29352951.

34. Gatta A, Dante A, Del Gaudio M, Pinna AD, Ravaioli M, Riganello I, et al. The use of prostaglandins in the immediate postsurgical liver transplant period. Transplant Proc. 2006;38:1092-1095.

35. Hamada T, Tsuchihashi S, Avanesyan A, Duarte S, Moore C, Busuttil RW, et al. Cyclooxygenase-2 deficiency enhances Th2 immune responses and impairs neutrophil recruitment in hepatic ischemia/reperfusion injury. J Immunol. 2008;180:1843-1853.

36. Duarte S, Kato H, Kuriyama N, Suko K, Ishikawa T-O, Busuttil RW, et al. Hepatic ischemia and reperfusion injury in the absence of myeloid cell-derived COX-2 in mice. PLoS ONE. 2014;9:e96913.

37. Nishizawa N, Ito Y, Eshima K, Ohkubo H, Kojo K, Inoue $\mathrm{T}$, et al. Inhibition of microsomal prostaglandin E synthase-1 facilitates liver repair after hepatic injury in

This article is protected by copyright. All rights reserved. 
mice. J Hepatol. 2018;69:110-120.

38. Cheng P, Wang F, Chen K, Shen M, Dai W, Xu L, et al. Hydrogen sulfide ameliorates ischemia/reperfusion-induced hepatitis by inhibiting apoptosis and autophagy pathways. Mediators Inflamm. 2014;2014:935251.

39. Francés DE, Motiño O, Agrá N, González-Rodríguez Á, Fernández-Álvarez A, Cucarella $\mathrm{C}$, et al. Hepatic cyclooxygenase-2 expression protects against diet-induced steatosis, obesity, and insulin resistance. Diabetes. 2015;64:1522-1531.

40. Rizzo MT. Cyclooxygenase-2 in oncogenesis. Clin Chim Acta. 2011;412:671-687.

41. Bejaoui M, Pantazi E, Calvo M, Folch-Puy E, Serafín A, Pasut G, et al. Polyethylene glycol preconditioning: an effective strategy to prevent liver ischemia reperfusion injury. Oxid Med Cell Longev. 2016;2016:9096549.

42. Ke B, Shen X-D, Zhang Y, Ji H, Gao F, Yue S, et al. KEAP1-NRF2 complex in ischemia-induced hepatocellular damage of mouse liver transplants. J Hepatol. 2013;59:1200-1207.

43. Kansanen E, Kivelä AM, Levonen A-L. Regulation of Nrf2-dependent gene expression by 15-deoxy-Delta12,14-prostaglandin J2. Free Radic Biol Med. 2009;47:1310-1317.

44. Pelissier-Rota MA, Pelosi L, Meresse P, Jacquier-Sarlin MR. Nicotine-induced cellular stresses and autophagy in human cancer colon cells: A supportive effect on cell homeostasis via up-regulation of Cox-2 and PGE(2) production. Int J Biochem Cell Biol. 2015;65:239-256.

45. Li T, Liu Y, Zhao J, Miao S, Xu Y, Liu K, et al. Aggravation of acute kidney injury by mPGES-2 down regulation is associated with autophagy inhibition and enhanced apoptosis. Sci Rep. 2017;7:10247.

46. Folch-Puy E, Panisello A, Oliva J, Lopez A, Castro Benítez C, Adam R, et al. Relevance of endoplasmic reticulum stress cell signaling in liver cold ischemia 
reperfusion injury. Int J Mol Sci. 2016;17.

47. Vilatoba M, Eckstein C, Bilbao G, Smyth CA, Jenkins S, Thompson JA, et al. Sodium 4-phenylbutyrate protects against liver ischemia reperfusion injury by inhibition of endoplasmic reticulum-stress mediated apoptosis. Surgery. 2005;138:342-351.

48. Martín-Sanz P, Boscá L, Olmedilla L, Perez-Peña J, Garutti I, Sanz J, et al. Presence of a nitric oxide synthase inhibitor in the graft efflux during reperfusion in human liver transplantation. Clin Transplant. 1999;13:221-230.

49. Garutti I, Sanz J, Olmedilla L, Tranche I, Vilchez A, Fernandez-Quero L, et al. Extravascular lung water and pulmonary vascular permeability index measured at the end of surgery are independent predictors of prolonged mechanical ventilation in patients undergoing liver transplantation. Anesth Analg. 2015;121:736-745.

This article is protected by copyright. All rights reserved. 


\section{FIGURE LEGENDS}

Figure 1. hCOX-2-Tg mice are protected against Ischemia/Reperfusion-induced liver damage.

Wt and hCOX-2-Tg mice were subjected to a warm hepatic ischemia for $90 \mathrm{~min}$ and to reperfusion (I/R) for 4 or $24 \mathrm{~h}$. (A) Representative Western blot of murine COX-1 and human COX-2 proteins in liver extracts. (B) $\mathrm{PGE}_{2}$ levels measured by EIA in liver from hCOX-2-Tg $v s$. Wt mice. $(\boldsymbol{C})$ Representative images of hematoxylin/eosin stained liver paraffin-embedded sections from Wt and hCOX-2-Tg mice after Sham or ischemia/reperfusion (I/R) at $4 \mathrm{~h}$ and 24 h. Necrotic areas are marked with $*$ and vascular congestion with $\rightarrow$. (D) Plasma levels of ALT, AST and LDH activities from Wt and hCOX-2-Tg mice. (E) Necrosis grade assessed by histological examination. Scores used were $0=$ none, $1=$ mild lesion $(0-20 \%$ of necrosis $)$, $2=$ moderate lesion $(20-40 \%$ of necrosis), $3=$ severe lesion (more than $40 \%$ of necrosis). Data are expressed as means \pm S.E. $(n=5-8$ per group). $* P<0.05$ vs. Wt-Sham; $\# P<0.05$ vs. Wt$\mathrm{I} / \mathrm{R}$.

Figure 2. Whole transcriptome sequencing reflects a differential gene expression profile in hCOX-2-Tg mice after I/R injury.

Total RNA was obtained from Wt and hCOX-2-Tg mice after $\mathrm{I} / \mathrm{R}$ at $4 \mathrm{~h}(\mathrm{n}=4$ in each experimental group). (A) Heat map of the two-way Hierarchical clustering (70 genes satisfying with $\mathrm{fc} \geq 2$ ) using Z-score for normalized value ( $\log 2$ based); (B) Scatter plot of expression level between two groups in hCOX-2 Tg mice vs Wt littermates. $(\boldsymbol{C})$ GeneEnrichment test based on Gene Ontology was conducted with significant gene list. The most significantly enriched biological process $(P<0.001$ by modified Fisher's exact test $)$ are shown.

This article is protected by copyright. All rights reserved. 
Figure 3. Analysis of pro-inflammatory cytokines and liver inflammation in Wt and hCOX-2-Tg mice after I/R injury.

(A) Hepatic mRNA levels of pro-inflammatory markers (Il-1b, Tnfa, Il-6, Nlrp3 and Il-10) in sham (Sh) or ischemia/reperfusion (I/R) conditions. (B) Plasmatic levels of IL-1 $\beta$, TNF- $\alpha$, and IL-6 were assessed by Luminex analysis. $(\boldsymbol{C})$ MPO activity measured in liver extract and (D) Representative immunohistochemistry for $\mathrm{Ly}_{6} \mathrm{G}^{+}$staining from $\mathrm{Wt}$ and hCOX-2-Tg mice after I/R. (E) Representative immunofluorescence merged images of p65 (red), albumin or CLEC4F (green) and DAPI (blue) from Wt and hCOX-2 Tg mice livers after I/R at $4 \mathrm{~h}$. Specific nuclear staining is marked with $\triangleright$. (F) Representative Western blots and densitometric analysis of NF- $\mathrm{KB}(\mathrm{p} 65)$ and $\mathrm{pIKB} \alpha$ protein levels in nuclear and cytosolic extracts, respectively. Data are expressed as means \pm S.E. ( $n=5-6$ per group). $* P<0.05$ vs. Wt-Sham; \#P<0.05 vs. Wt-I/R.

Figure 4. Hepatic hCOX-2 expression leads to increased antioxidant defenses and decreased I/R-derived oxidative stress.

(A) mRNA levels of Nrf2 and antioxidant genes (Hmoxl, Gpx, Gsr, Sodl and Sod2) in liver extracts in sham $(\mathrm{Sh})$ or ischemia/reperfusion (I/R) at $4 \mathrm{~h}$. (B) Analysis of NRF2 nuclear expression levels in liver extracts from $\mathrm{Wt}$ and hCOX-2-Tg mice after I/R at $4 \mathrm{~h}$. (C) Representative merged images of NRF2 (red), albumin or CLEC4F (green) and DAPI (blue) immunostaining in liver from $\mathrm{Wt}$ and hCOX-2-Tg mice after I/R at $4 \mathrm{~h}$. Specific nuclear staining is marked with $D$. (D) MnSOD activity assessed by NBT reduction inhibition assay in native polyacrylamide gel electrophoresis. $(\boldsymbol{E})$ Representative images of dihydroethidium staining in liver sections in liver from $\mathrm{Wt}$ and $\mathrm{hCOX}-2-\mathrm{Tg}$ mice in sham (Sh) or ischemia/reperfusion (I/R) at 4 h. $\quad(\boldsymbol{F})$ Total glutathione content and Oxidized glutathione/Total glutathione ratio (GSSG/GSHt). Data are expressed as means \pm S.E. $(n=4-8$ mice per group). ${ }^{*} P<0.05$ vs. Wt-Sham; $\# P<0.05$ vs. Wt-I/R.

This article is protected by copyright. All rights reserved. 
Figure 5. COX-2 expression improves autophagy and decreases ER stress markers in liver after I/R injury.

(A-C) Representative Western blots and densitometric analysis of p62 (A), LC3II (B), and ATG7 $(\boldsymbol{C})$ protein levels in sham $(\mathrm{Sh})$ or ischemia/reperfusion $(\mathrm{I} / \mathrm{R})$ conditions. (D) Transmission electron microscopic micrographs of livers from $\mathrm{Wt}$ and hCOX-2-Tg mice after $4 \mathrm{~h} \mathrm{I} / \mathrm{R}$. Original magnification x4000. Autophagosomes are marked with $\mathbf{v} . \mathrm{n}$ means nucleus. $(\boldsymbol{E}-\boldsymbol{F})$ Representative Western blots and densitometric analysis of GPR78 (E), and CHOP $(\boldsymbol{F})$ protein levels. Data are expressed as means \pm S.E. ( $n=4-6$ per group). $* P<0.05 v s$. Wt-Sham; $\# P<0.05$ vs. Wt-I/R.

Figure 6. Ischemic preconditioning induces Cox-2 mRNA and PGE production attenuating Ischemia/Reperfusion-induced liver damage.

(A) Representative images of hematoxylin/eosin stained liver paraffin-embedded sections from Wt, Wt PC, DFU-treated Wt PC (Wt PC DFU), hCOX-2-Tg, and DFU-treated hCOX-2Tg mice (hCOX-2-Tg DFU) after I/R at $4 \mathrm{~h}$. Necrotic areas are marked with * and vascular congestion with $\boldsymbol{G}$. (B) Necrosis grade assessed by histological examination. Scores used were $0=$ none, $1=$ mild lesion $(0-20 \%$ of necrosis), $2=$ moderate lesion $(20-40 \%$ of necrosis), $3=$ severe lesion (more than $40 \%$ of necrosis). (C) Plasma levels of ALT, AST and LDH activities. (D) PGE $_{2}$ levels were measured by EIA in Wt, Wt PC, DFU-treated Wt PC, hCOX2-Tg, and DFU-treated hCOX-2-Tg mice after I/R at $4 \mathrm{~h}(\boldsymbol{E})$ Mouse (m) endogenous Cox-2 mRNA and protein levels in Wt and Wt PC mice after I/R at $4 \mathrm{~h}$. (F) Representative immunofluorescence merged images of mCOX-2 (red), albumin or CLEC4F (green) and DAPI (blue) from Wt and Wt PC mice livers after I/R at $4 \mathrm{~h}$. Hepatocyte staining is marked with $\triangleright$. Data are expressed as means \pm S.E. ( $n=5-8$ per group). $* P<0.05$ vs. Wt-Sham, $\# P<0.05$ vs. Wt-I/R; $\S P<0.05$ vs. Wt-PC; $\uparrow P<0.05$ vs. hCOX-2-Tg-I/R.

This article is protected by copyright. All rights reserved. 
Figure 7. Analysis of pro-inflammatory cytokines and antioxidant genes after Ischemic preconditioning.

(A) mRNA levels of pro-inflammatory markers (Il-1b, Il-6, Tnfa, Nlrp3, and Il-10) in liver from Wt, Wt PC, hCOX-2 and DFU-treated hCOX-2 mice in sham (Sh) or ischemia/reperfusion (I/R) conditions at 4 h. (B) mRNA levels of $N r f 2$ and its target genes (Hmoxl, Gpx, Gsr, Sodl and Sod2) in liver extracts from Wt, Wt PC, hCOX-2 and DFUtreated hCOX-2 mice after I/R at $4 \mathrm{~h}$. Data are expressed as means \pm S.E. ( $n=5-8$ per group). $* P<0.05$ vs. Wt-Sham; $\# P<0.05$ vs. Wt-I/R; $† P<0.05$ vs. hCOX-2-Tg-I/R.

Figure 8. Liver graft function respect to the level of $P G E_{2}$ measured in R60 plasma. (A) $\mathrm{PGE}_{2}$ levels measured by EIA were analyzed in arterial plasma obtained from 63 patients receiving LT collected 60 min after reperfusion. Graft function was evaluated during the first 3 days after LT; Mann-Whitney rank-sum test were used to analyzed the relationship between $\mathrm{PGE}_{2}$ levels and early graft dysfunction: NO, good graft function (grades I or II); YES, poor graft function (grades III or IV), (* $P=0,026) ;(\boldsymbol{B}-\boldsymbol{C})$ To assess a significant relationship between $\mathrm{PGE}_{2}$ levels and ischemia time $(\boldsymbol{B})$ or length of hospital stay $(\boldsymbol{C})$, Spearman's correlation was calculated, showing a significant inverse correlation between both parameters.

This article is protected by copyright. All rights reserved. 
Figure 1

A

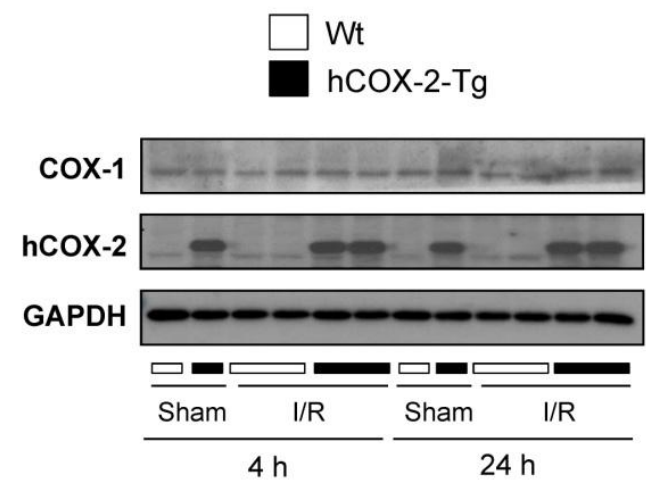

C

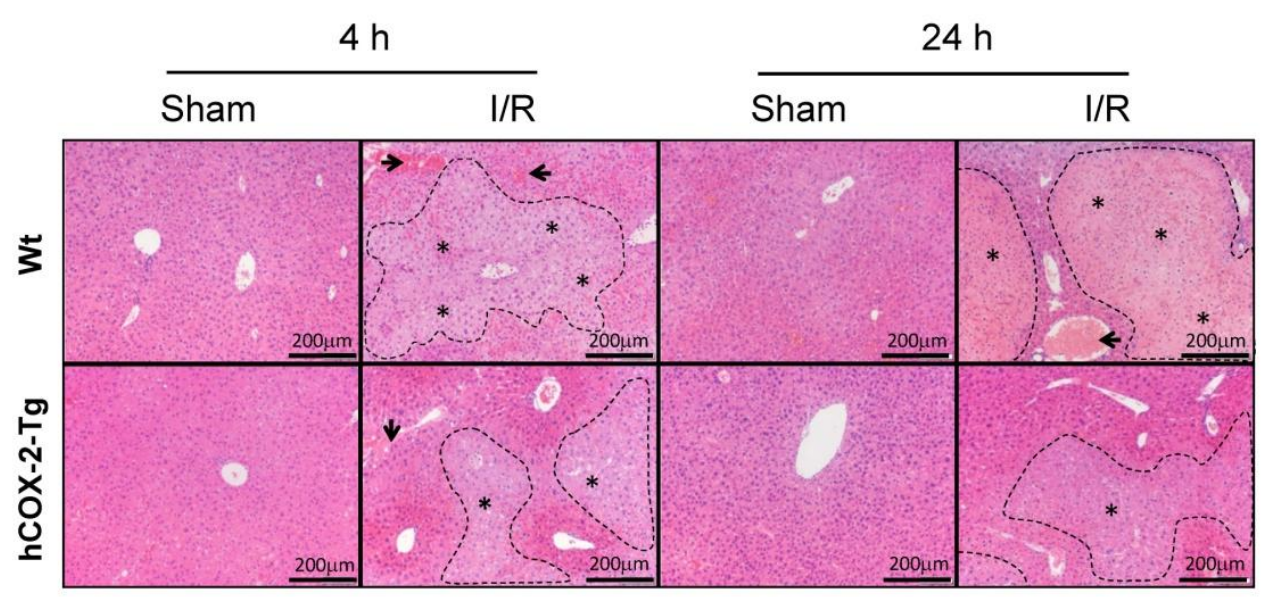

B

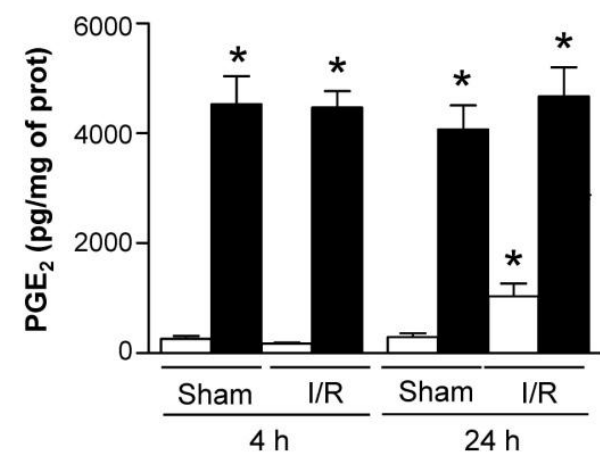

E

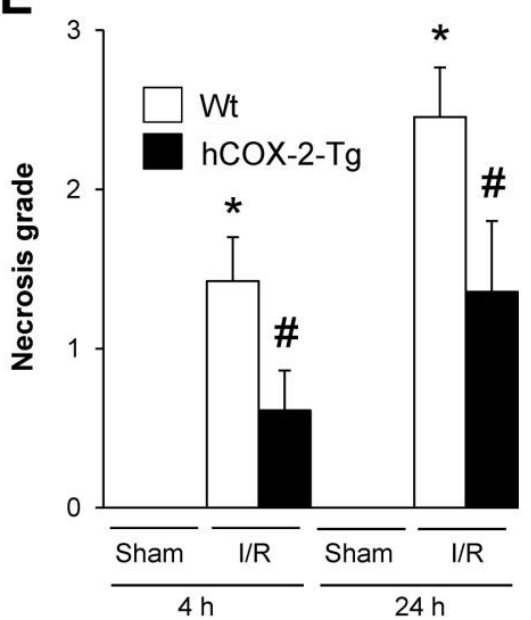

This article is protected by copyright. All rights reserved. 


\section{Figure 2}

A

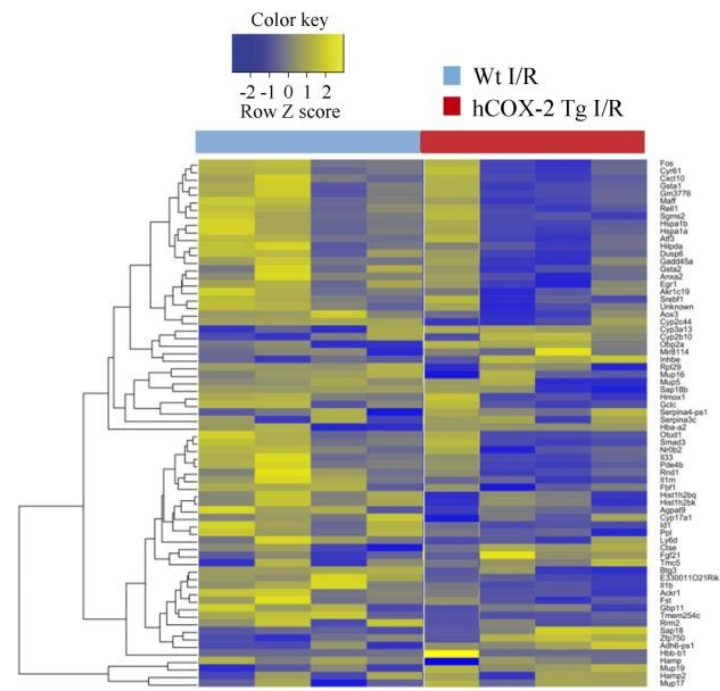

B

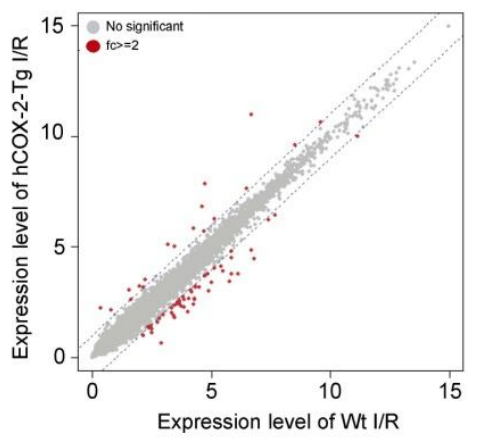

C

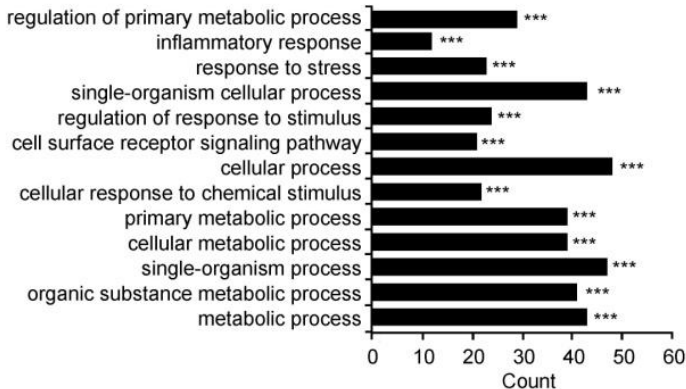

This article is protected by copyright. All rights reserved. 


\section{Figure 3}
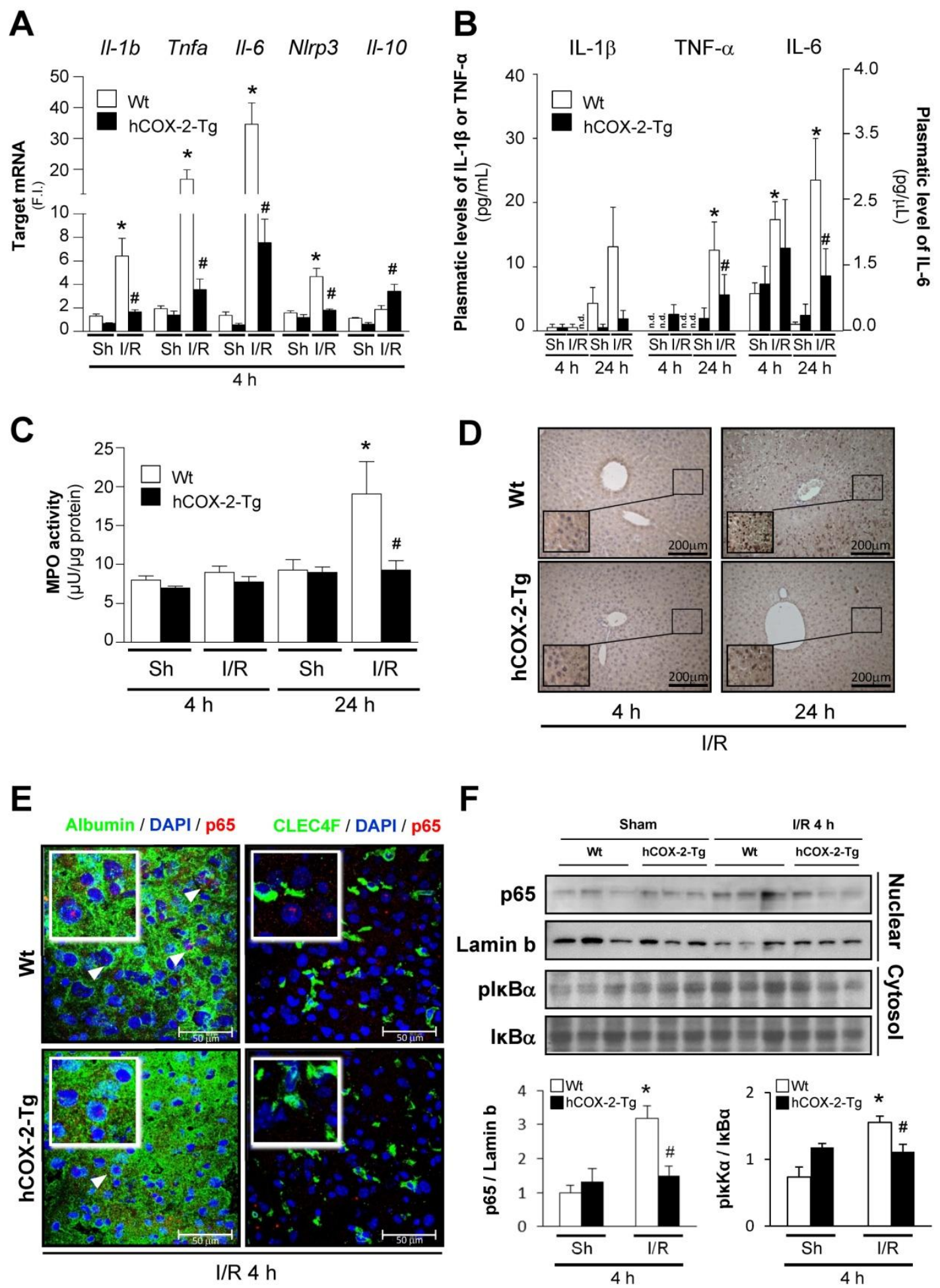

This article is protected by copyright. All rights reserved. 
Figure 4

A

B

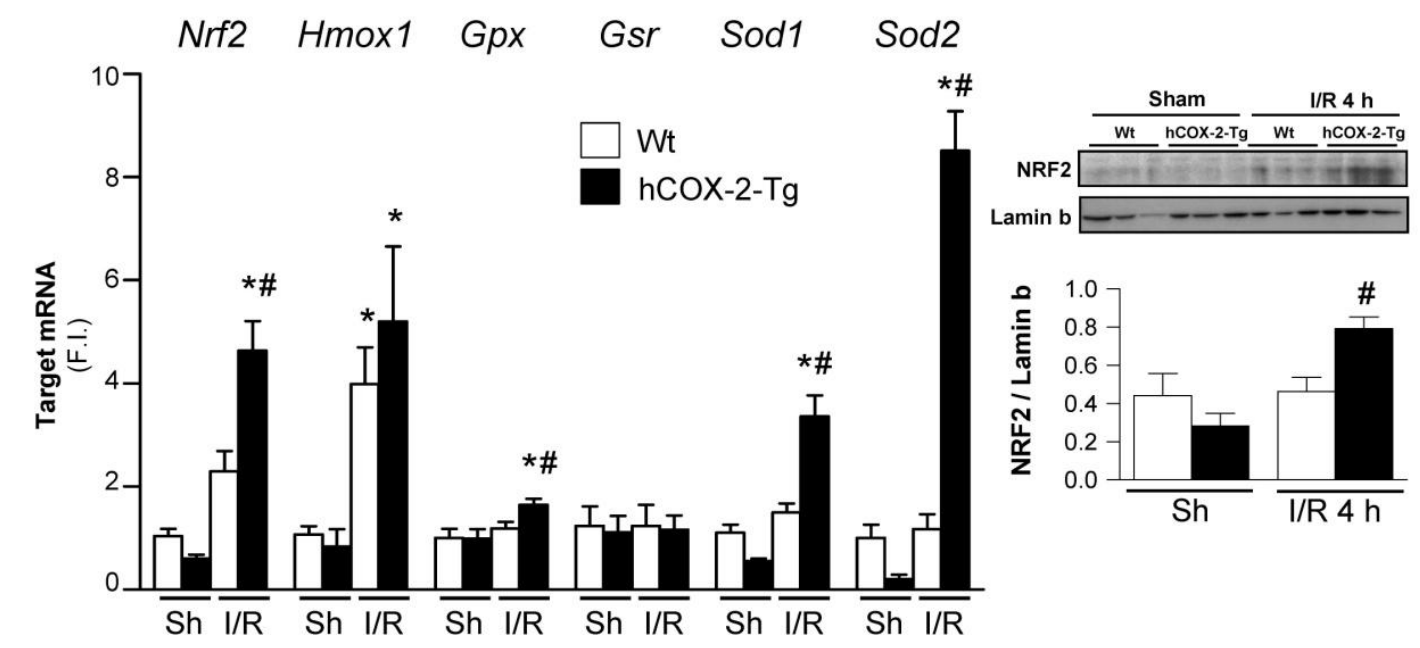

C ALBUIMIN/DAPI/NRF2 CLEC4FIDAPI/NRF2

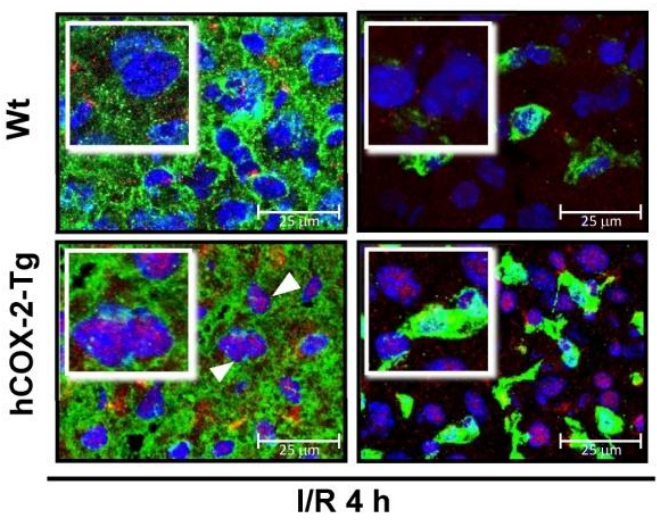

D
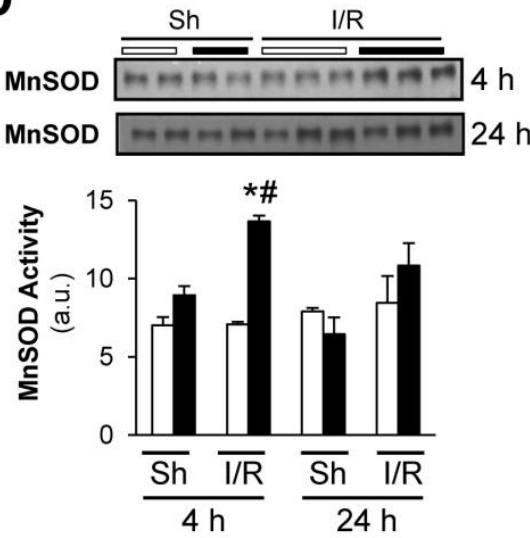

E

$\mathbf{F}$
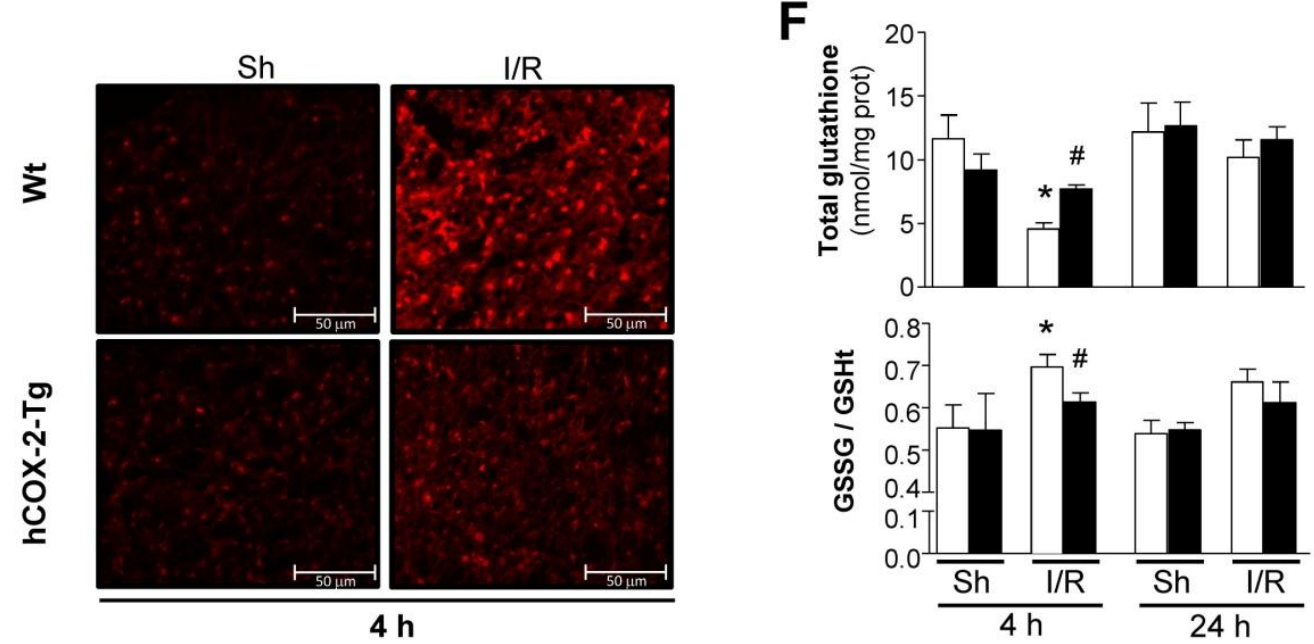

This article is protected by copyright. All rights reserved. 
Figure 5

$\square$ Wt $\square$ hCOX-2-Tg

A

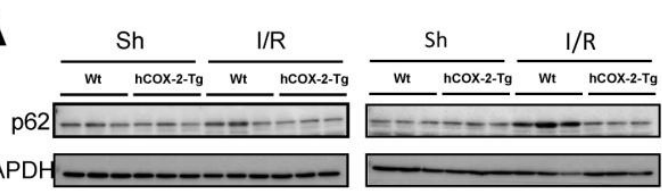

B

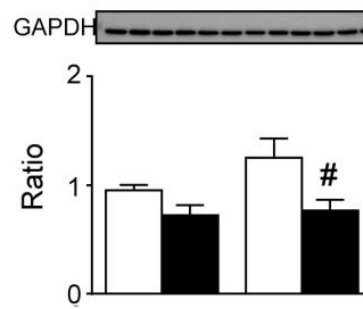

$4 \mathrm{~h}$

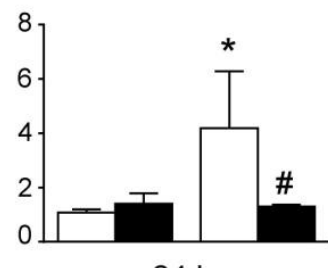

$24 \mathrm{~h}$
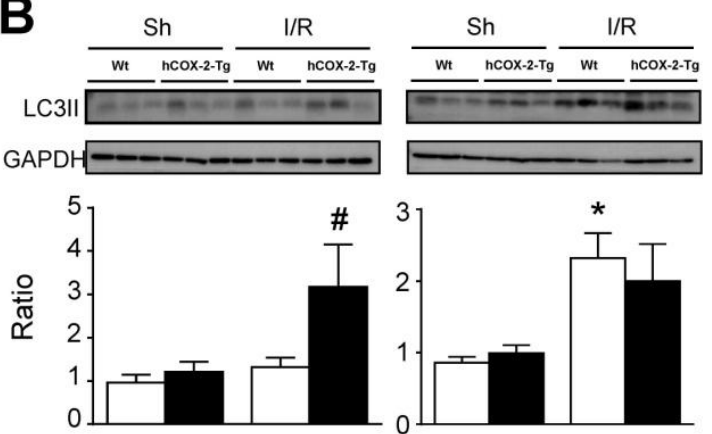

$4 \mathrm{~h}$

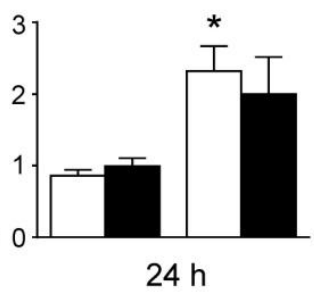

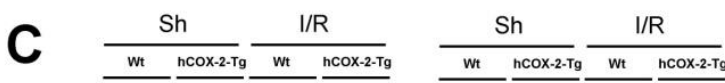
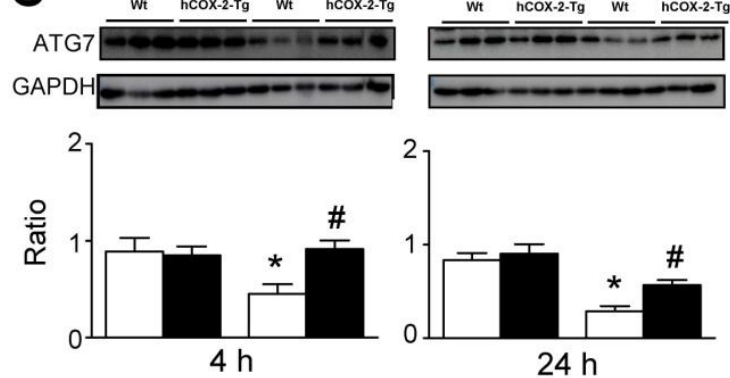

D

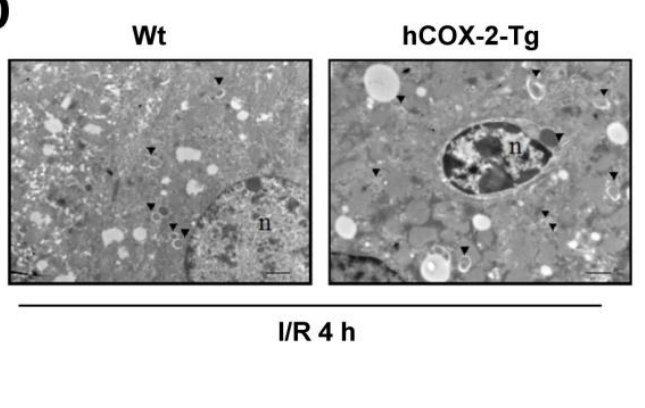

E

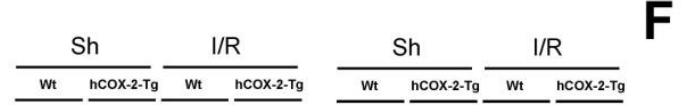

$\mathbf{F}$

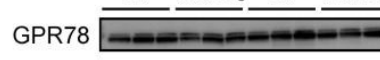

GAPDH

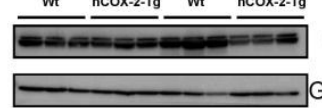

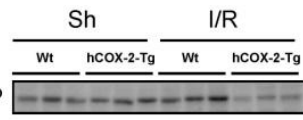

GAPDH
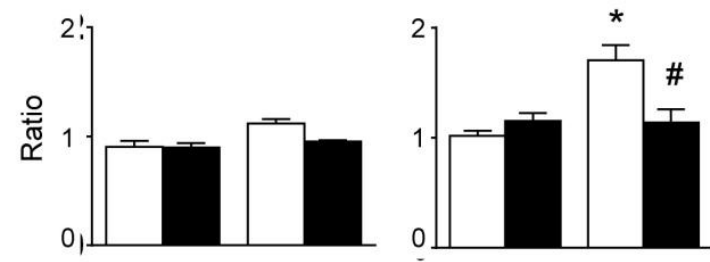

$24 \mathrm{~h}$

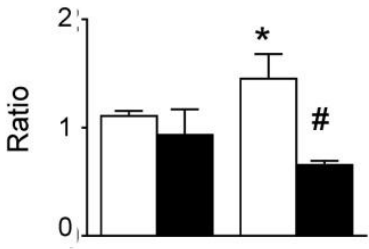

$4 \mathrm{~h}$
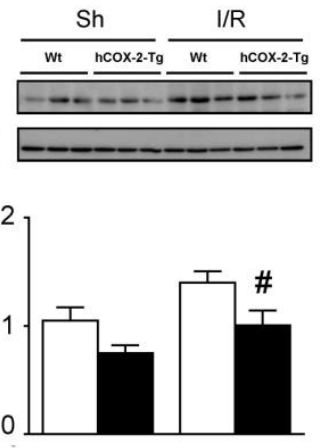

$24 \mathrm{~h}$

This article is protected by copyright. All rights reserved. 
Figure 6

A
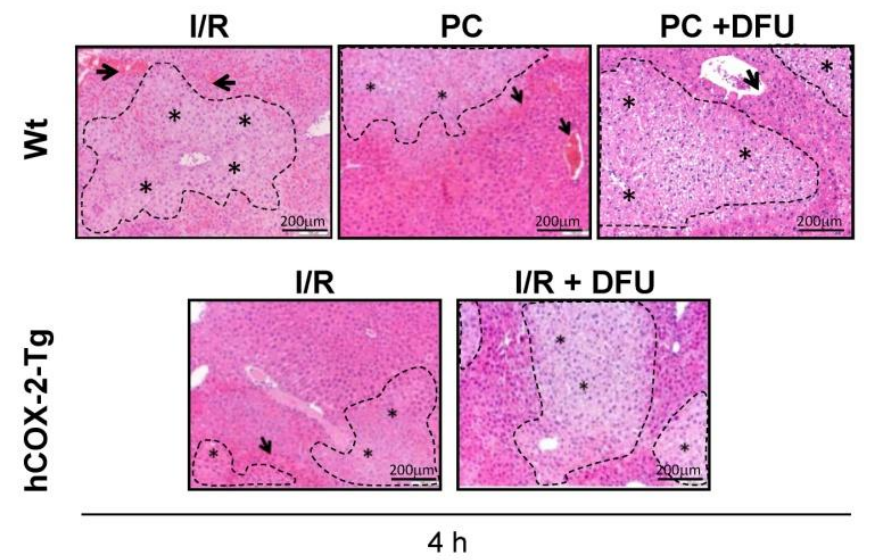

C

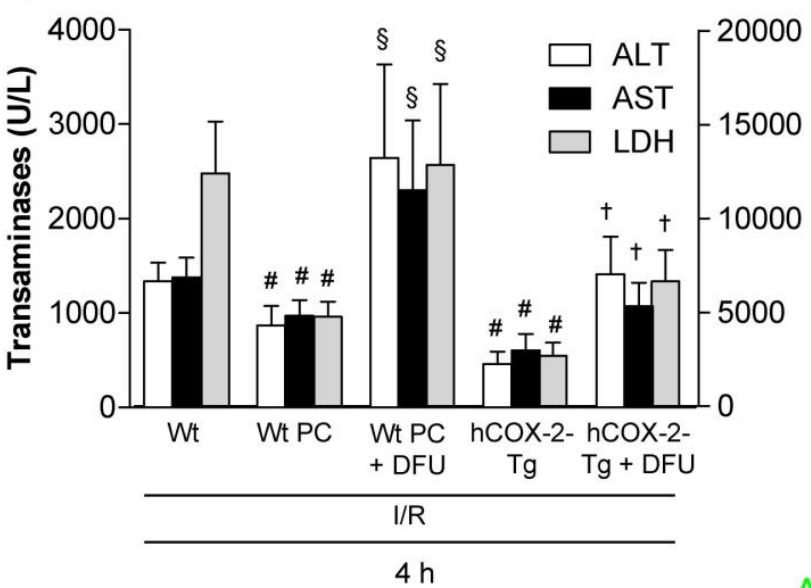

E

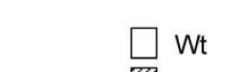

Wt PC

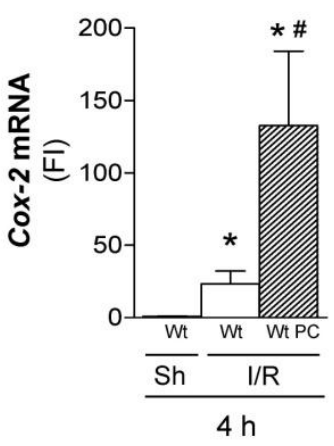

F

$\frac{W t}{W t} W_{t} P C$ GAPDH

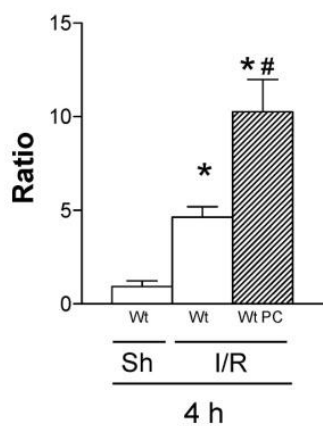

$\square \mathrm{W}$

Wt PC

$\mathrm{hCOX}-2-\mathrm{Tg}$

Wt PC + DFU

B

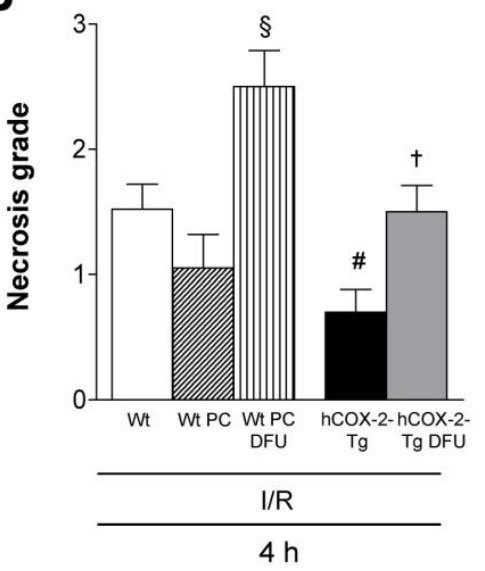

D

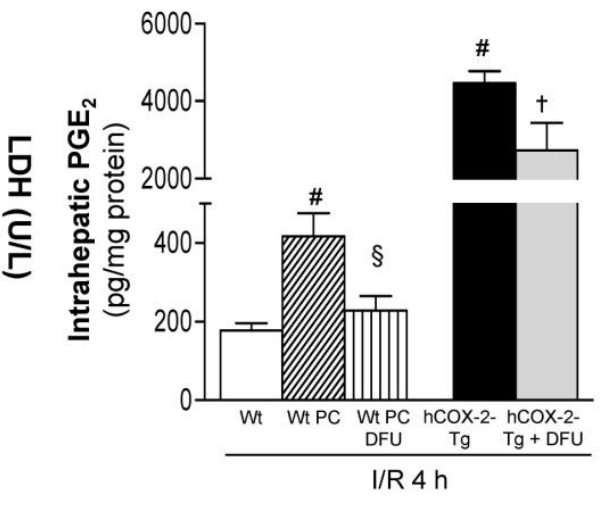

ALBUMIN/DAPI/mCOX-2CLEC4F/DAPI/mCOX-2
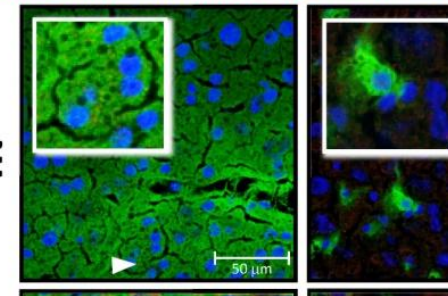

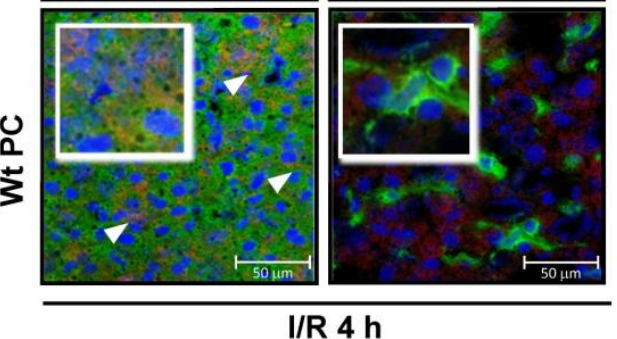

This article is protected by copyright. All rights reserved. 
Figure 7
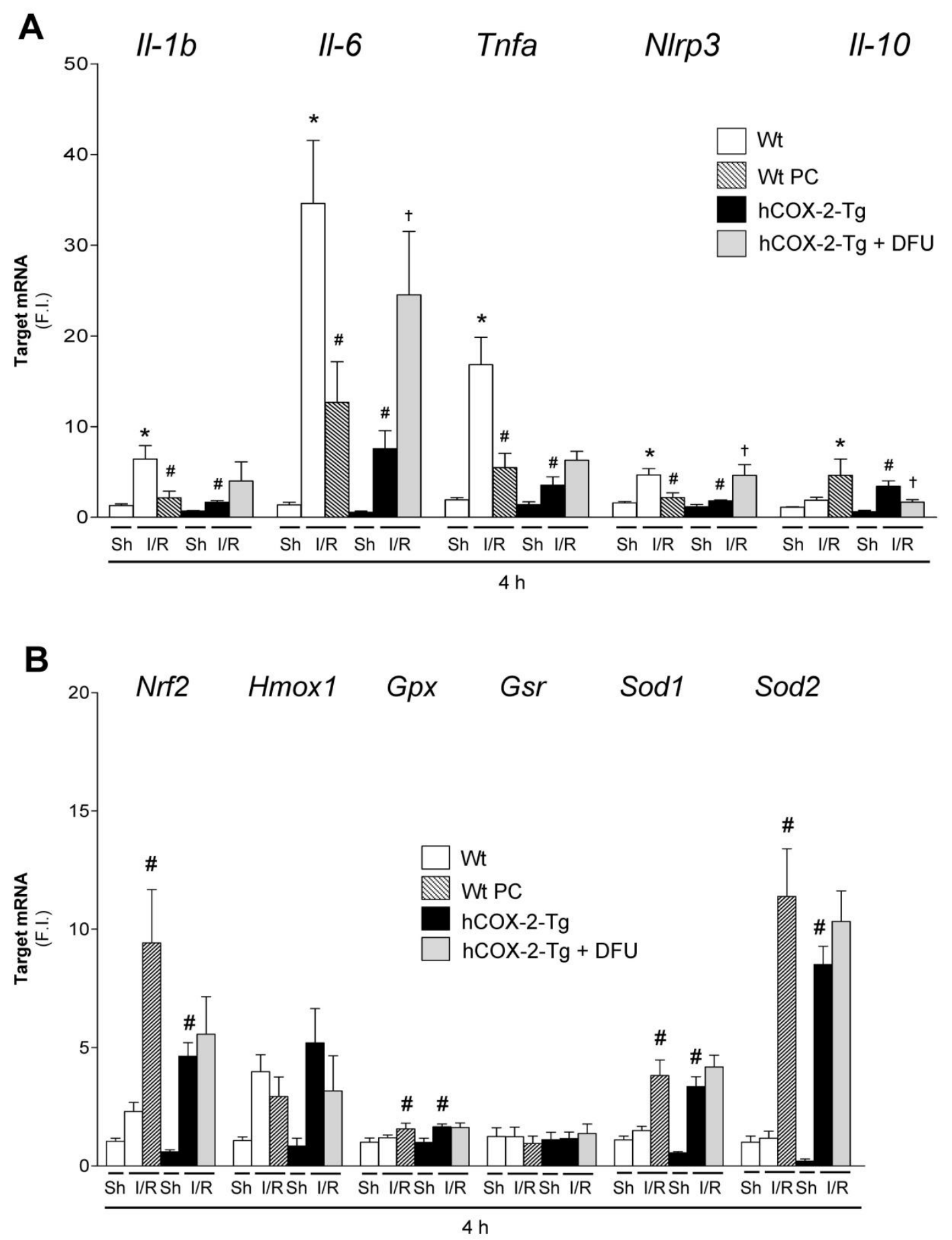

This article is protected by copyright. All rights reserved. 


\section{Figure 8}

A

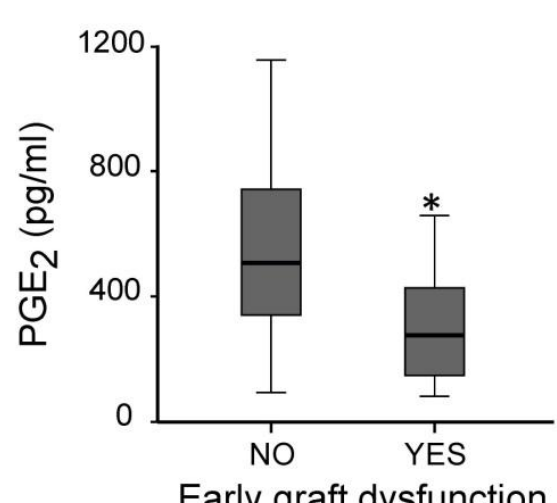

B

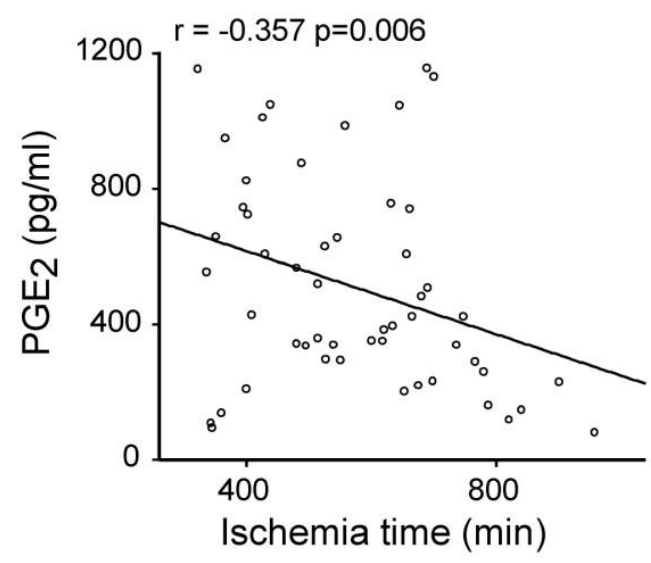

C

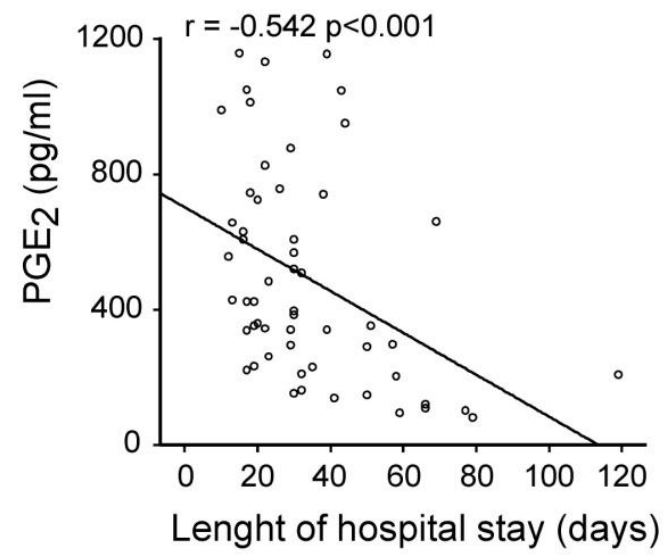

This article is protected by copyright. All rights reserved. 